\title{
TDP-43 in the muscles: friend or foe?
}

myo-granules
formed
during tissue
regeneration
might seed
the formation
of TDP-43
aggregates

A typical histological feature of inclusion body myositis (IBM) is cytoplasmic aggregation of the RNA binding protein TAR DNA-binding protein 43 (TDP-43) in the skeletal muscles. Although typically thought of as a pathogenic feature, new findings published in Nature suggest that assemblies of TDP-43 have a beneficial function in muscle regeneration.

Muscle aggregation of TDP-43 is a feature of other rimmed vacuole myopathies (including a hereditary inclusion body myopathy, oculopharyngeal muscular dystrophy and distal myopathies with rimmed vacuoles), and aggregation in the neurons is also a feature of the neurodegenerative disorders amyotrophic lateral sclerosis and frontotemporal lobar degeneration.

"The combination of TDP-43 aggregation properties in multiple diseases, the accessibility of muscle as a long-lived, complex tissue prone to degenerative diseases that we could study, and the suggestions that TDP-43 played some important, but undetermined, function in muscle formation and/or regeneration convinced us to pursue TDP-43 function," says Roy Parker, one of the corresponding authors of the new study.

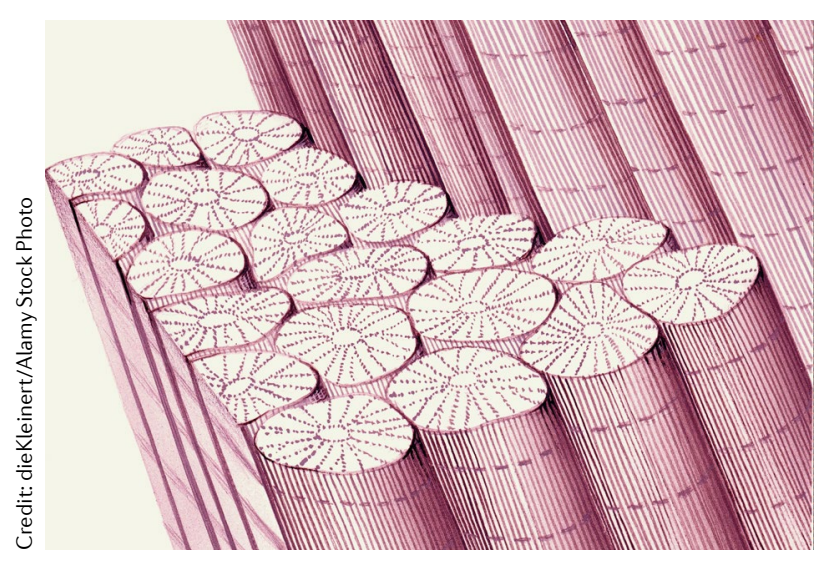

The researchers initially studied the subcellular distribution of TDP-43 in cultured primary mouse myoblasts and $\mathrm{C} 2 \mathrm{C} 12$ myoblasts, a mouse muscle cell line. As expected, TDP-43 predominantly localized to the nucleus in myoblasts; however, TDP-43 localization shifted to the cytoplasm following myoblast differentiation into myotubes. In vivo, TDP-43 localized to the nucleus in uninjured tibialis anterior muscle but was also found in the cytoplasm at sites of newly forming sarcomeres in myofibres that were regenerating after chemically induced damage.

"We found TDP-43 assembles into an undiscovered RNA-protein assembly (referred to as a myogranule)," explains Thomas Vogler, a coauthor of the study. "The myo-granule has amyloid-like properties associated with disease states and thus, our findings are of notable interest because TDP-43containing amyloid-like assemblies seem to be involved in normal muscle rebuilding after an injury".

In addition to mRNA, these myo-granules were enriched for proteins involved in RNA localization and translation. The set of mRNAs bound by TDP-43 during muscle formation differed from that in undifferentiated myoblasts and included mRNA transcripts associated with the sarcomere, suggesting a different function for TDP-43 during myogenesis that involves the regulation of structural mRNAs.

In vivo, deletion of one copy of the gene encoding TDP-43 (Tardbp) in the muscle stem cells of mice resulted in smaller myofibres after injury (compared with wild-type mice). As the size and number of muscle stem cells before injury were unaffected, the findings suggest a role of TDP-43 in skeletal muscle regeneration.

In $V c p$ mutant mice, a model of a hereditary inclusion body myopathy caused by mutations in valosin-containing protein (VCP), levels of TDP-43-containing myo-granules were higher than in wild-type mice and correlated with muscle regeneration. The investigators speculate that myogranules formed during tissue regeneration might seed the formation of TDP-43 aggregates in disease, linking the biological and potential pathological function of TDP-43.

Indeed, myo-granules from C2C12 myotubes could transition into higher-order amyloid-like fibrous structures in vitro; these aggregates were similar in appearance to that previously reported for TDP-43 amyloid fibres, and their formation was exacerbated by the addition of recombinant TDP-43.

"If disruption of mechanisms involved in the formation or dissolution of TDP-43 aggregates contributes to human disease (still an unresolved issue), then perturbing the formation of myogranules (or similar assemblies in neurons) might lead to new therapeutic approaches to limit TDP-43 aggregation," says co-author Joshua Wheeler.

"We would like to determine the genetic and biochemical basis of the amyloid-like state of TDP-43," reveals the co-corresponding author Bradley Olwin. "In doing so, we can test the biological importance of forming this amyloid-like assembly, as well as its contribution to disease."

Jessica McHugh

ORIGINAL ARTICLE Vogler, T. O. et al. TDP-43 and RNA form amyloid-like myo-granules in regenerating muscle. Nature 563, 508-513 (2018) 